\title{
Schweizerischer Impfplan 2009
}

Bundesamt für Gesundheit, Eidgenössische Kommission für Impffragen

1 Bundesamt für Gesundheit, Eidgenössische Kommission für Impffragen (EKIF). Schweizerischer Impfplan 2009. Richtlinien und Empfehlungen. Bern: BAG; 2009.

2 Bundesamt für Gesundheit, Eidgenössische Kommission für Impffragen, Schweizerische Gesellschaft für Neonatologie, Schweizerische Gesellschaft für Pädiatrie. Impfung von Frühgeborenen. Richtlinien und Empfehlungen. Bern: BAG; 2009.

3 Bundesamt für Gesundheit. Rotavirus-Impfung: keine Aufnahme in den Schweizerischen Impfplan. BAG Bulletin. 2008;(28):492-5

4 Verordnung des Eidgenössischen Departements des Innern über Leistungen in der obligatorischen Krankenpflegeversicherung (Krankenpflegeleistungsverordnung, KLV). Änderung vom 10. Dezember 2008 (www.bag.admin.ch, Krankenversicherung, Rechts- und Vollzugsgrundlagen).
Der Schweizerische Impfplan 2009 (Richtlinien und Empfehlungen) wurde im Februar publiziert [1]. Er kann von den Internetseiten des Bundesamtes für Gesundheit und von InfoVac heruntergeladen werden: www.sichimpfen.ch oder www. infovac.ch.

\section{Neuerungen und Anpassungen 2009}

\section{Empfehlungen für die Nachholimpfungen bei ungenügend geimpften Kindern und Erwachsenen [1]}

Empfehlungen für Nachholimpfungen können sehr komplex sein. Verschiedene neue Tabellen dienen dabei als Entscheidungshilfe. Der Impfplan 2009 wurde zudem ergänzt durch neue Empfehlungen für die Nachholimpfungen gegen Keuchhusten bei nicht- oder ungenügend geimpften Kindern und Jugendlichen.

\section{Empfohlene Impfungen für Risikogruppen: Frühgeborene [2]}

Kinder, die vor der 33. Gestationswoche oder mit einem Geburtsgewicht von $<1500 \mathrm{~g}$ geboren werden, sind besonders anfällig für Infektionen und stellen daher eine Risikogruppe dar, für die spezifische Impfempfehlungen angezeigt sind: gestraffter Impfplan für die DTP $\mathrm{a}$-Hib-IPV- $( \pm \mathrm{HB}-)-$ Impfung mit 2, 3 und 4 Monaten und Auffrischimpfung mit 12 Monaten, MMR-Impfung mit 9 und 12 Monaten, Grippeimpfung ab 6. Monat während der beiden ersten Influenzasaisons. Dieser Impfplan wird ergänzt durch spezifische Empfehlungen für die Familienangehörigen.

\section{Impfungen ohne Empfehlungen: \\ Rotaviren [3]}

Das BAG und die Eidgenössische Kommission für Impffragen (EKIF) haben entschieden, die Rotavirenimpfung zum gegenwärtigen Zeitpunkt nicht in den Schweizerischen Impfplan aufzunehmen. Diese Empfehlung wird erneut evaluiert, falls sich Änderungen ergeben, die das Kosten-Nutzen-Verhältnis günstig beeinflussen sollten.

\section{Kostenübernahme der Pneumokokken- impfung bei Personen ab 65 Jahren [4]} Die Kosten für die Pneumokokkenimpfung für Personen ab 65 Jahren werden seit dem 1. Januar 2009 von der obligatorischen Krankenpflegeversicherung übernommen gemäss folgendem Impfschema: eine Dosis mit dem Polysaccharidimpfstoff.

\section{Tabelle}

Empfohlene Basisimpfungen und ergänzende Impfungen (EKIF/BAG).

\begin{tabular}{|c|c|c|c|c|c|c|c|c|c|c|c|c|}
\hline \multirow[b]{2}{*}{ Alter $^{1}$} & \multicolumn{8}{|c|}{ Basisimpfungen } & \multicolumn{4}{|c|}{ Ergänzende Impfungen } \\
\hline & DTP & Polio & Hib & $\mathrm{HB}^{6}$ & MMR & HPV & VZV & Influenza & $\begin{array}{l}\text { Pneumo- } \\
\text { kokken }\end{array}$ & $\begin{array}{l}\text { Pneumo- } \\
\text { kokken }\end{array}$ & $\begin{array}{l}\text { Meningo- } \\
\text { kokken C }\end{array}$ & HPV \\
\hline Geburt & & & & 7 & & & & & & & & \\
\hline 2 Monate & DTP $_{a}$ & IPV & $\mathrm{Hib}^{4}$ & 8 & & & & & & PCV 717,18 & & \\
\hline 4 Monate & DTP $_{a}$ & IPV & $\mathrm{Hib}$ & 8 & & & & & & PCV7 & & \\
\hline 6 Monate & DTP $_{a}$ & IPV & $\mathrm{Hib}$ & 8 & & & & & & & & \\
\hline 12 Monate & & & & & MMR & & & & & PCV7 ${ }^{18}$ & & \\
\hline 12-15 Monate & & & & & & & & & & & $\mathrm{MCV}-\mathrm{C}^{19}$ & \\
\hline 15-24 Monate & DTPa $_{a}$ & IPV & $\mathrm{Hib}^{5}$ & 8 & MMR & & & & & & & \\
\hline 4-7 Jahre & DTPa $_{a}$ & IPV & & & 10 & & & & & & & \\
\hline 11-14/15 Jahre & $\mathrm{dT} \mathrm{p}_{\mathrm{a}} / \mathrm{dT^{2 }}$ & 3 & & $\mathrm{HBV}^{9}$ & 10 & $\mathrm{HPV}^{12}$ & $\mathrm{VZV}^{14}$ & & & & $\mathrm{MCV}-\mathrm{C}^{20}$ & \\
\hline Erwachsene & dT & 3 & & 9 & 11 & 13 & 14 & 15 & 16 & & & $\mathrm{HPV}^{21}$ \\
\hline
\end{tabular}

1-21: vgl. Originaldokument auf www.sichimpfen.ch oder www.infovac.ch. 